SLAC-PUB-9031

BABAR-PROC-01/68

October, 2001

\title{
Observation of $\mathrm{CP}$ violation in the $B^{0}$ system
}

\author{
Christos Touramanis \\ Department of Physics \\ Oliver Lodge Laboratory, University of Liverpool \\ L69 7ZE, Liverpool, U.K. \\ (for the BABAR Collaboration)
}

\begin{abstract}
The BABAR detector, operating at energies near the $\Upsilon(4 S)$ resonance at the PEP-II asymmetric $B$ Factory at SLAC, has collected a sample of 32 million $B \bar{B}$ pairs by May 2001. A study of timedependent $C P$-violating asymmetries in events where one neutral $B$ meson is fully reconstructed in a final state containing charmonium has resulted in the measurement $\sin 2 \beta=0.59 \pm 0.14$ (stat) \pm 0.05 (syst), which establishes $C P$ violation in the $B^{0}$ meson system at the $4 \sigma$ level. $B$ lifetime and mixing measurements from a sub-sample of 23 million $B \bar{B}$ pairs are also presented.
\end{abstract}

Invited talk presented at the International Europhysics Conference on HEP

(HEP2001)

12-18 July 2001, Budapest, Hungary

Stanford Linear Accelerator Center, Stanford University, Stanford, CA 94309

Work supported by the U.K. Particle Physics and Astronomy Research Council, Advanced Research Fellowship GR/L04177 and by Department of Energy contract DE-AC03-76SF00515. 


\section{Introduction}

Since its discovery in 1964 in the decays of $K_{L}^{0}$ mesons [1] $C P$ violation has been the subject of many experiments and the motivation for many theoretical developments in particle physics. The three-generation Standard Model accommodates $C P$ violation through the presence of a non-zero imaginary phase in the Cabibbo-Kobayashi-Maskawa (CKM) quark mixing matrix [2]. The result presented here establishes for the first time the existence of $C P$ violation outside the neutral Kaon system.

The primary goal of the BABAR experiment at PEP-II is to perform stringent tests of the Standard Model by over-constraining the Unitarity Triangle through $C P$ violation measurements (angles $\alpha, \beta$ and $\gamma$ ) and the determination of its sides $\left(\left|V_{u b}\right|,\left|V_{c b}\right|\right.$ in semileptonic $B$ decays and $\left|V_{t d}\right|$ in $B^{0} \bar{B}^{0}$ mixing).

\section{PEP-II}

The PEP-II $B$ Factory [3] is an $e^{+} e^{-}$colliding beam storage ring complex at SLAC designed to produce a luminosity of $3 \times 10^{33} \mathrm{~cm}^{-2} \mathrm{~s}^{-1}$ at a center-of-mass energy of $10.58 \mathrm{GeV}(\Upsilon(4 S)$ resonance). At the $\Upsilon(4 S)$ resonance $B$ mesons can only be produced as $B^{+} B^{-}$or coherent $B^{0} \bar{B}^{0}$ pairs. The time evolution of a coherent $B^{0} \bar{B}^{0}$ pair is coupled in such a way that the $C P$ or flavor of one $B$ at decay time $t_{1}$ can be described as a function of the other $B$ flavor at its decay time $t_{2}$ and the signed time difference $\Delta t=t_{1}-t_{2}$. The machine has asymmetric energy beams $(9.0 \mathrm{GeV}$ electrons on $3.1 \mathrm{GeV}$ positrons), corresponding to a center-of-mass boost of $\beta \gamma=0.56$. An average separation of $\beta \gamma \mathrm{c} \tau \approx 250 \mu \mathrm{m}$ between the two $B$ meson decay vertices allows the measurement of time-dependent decay rate asymmetries. PEP-II has exceeded its design luminosity by $30 \%$ while BABAR, with a logging efficiency of $>95 \%$, has been accumulating data at daily rates up to $260 \mathrm{pb}^{-1}$.

\section{$3 \quad B A B A R$}

A detailed description of the detector and its performance can be found in [3]. The volume within the 1.5T BABAR superconducting solenoid contains a five layer silicon strip vertex detector (SVT), a central drift chamber with a helium-based gas mixture (DCH), a quartz-bar Cherenkov radiation detector (DIRC) and a CsI(Tl) crystal electromagnetic calorimeter (EMC). Two layers of cylindrical resistive plate counters (RPCs) are located between the barrel calorimeter and the magnet cryostat. The instrumented flux return (IFR) outside the cryostat is composed of 18 layers of radially increasing thickness steel, instrumented with 19 layers of planar RPCs in the barrel and 18 in the endcaps which provide muon and neutral hadron identification.

The SVT has a typical single hit resolution of $15 \mu \mathrm{m}$ in $z$ and $97 \%$ efficiency, while fully reconstructed single $B$ decay vertex resolution in $z$ is $50 \mu \mathrm{m}$. Charged particle tracking using the SVT and DCH achieves a resolution of $\left(\sigma\left(p_{T} / p_{T}\right)\right)^{2}=\left(0.0015 p_{T}\right)^{2}+(0.0045)^{2}$, where $p_{T}$ is the transverse momentum in $\mathrm{GeV} / c$. Photons are reconstructed in the EMC, yielding mass resolutions of $6.9 \mathrm{MeV} / c^{2}$ for $\pi^{0} \rightarrow \gamma \gamma$ and $10 \mathrm{MeV} / c^{2}$ for $K_{S}^{0} \rightarrow \pi^{0} \pi^{0}$.

Leptons and hadrons are identified using a combination of measurements from all the BABAR components, including energy loss $\mathrm{d} E / \mathrm{d} x$ in the $\mathrm{DCH}$ and in the SVT. Electron identification is mainly based on deposited energy and shower characteristics in the EMC, while muons are identified in the IFR and confirmed by their minimum ionizing signal in the EMC. Excellent kaon 
identification in the barrel region is provided by the DIRC, which achieves a $K-\pi$ separation of $>3.4 \sigma$ in the range $0.25-3.0 \mathrm{GeV} / c$.

\section{Mixing and $C P$ violation with dilepton events}

The mass difference $\Delta m_{d}$ between the two mass eigenstates of the neutral $B$ system (sensitive to $\left.\left|V_{t d}\right|\right)$ is measured by comparing the rate of neutral $B$ meson pairs decaying with the same $b$ quark flavor to the rate of decays with the opposite flavor sign. At the $\Upsilon(4 S)$ this time dependent asymmetry gives direct access to $\Delta m_{d}$ :

$$
A_{\Delta m_{d}}(\Delta t)=\frac{N\left(B^{0} \bar{B}^{0}\right)(\Delta t)-\left(N\left(B^{0} B^{0}\right)(\Delta t)+N\left(\bar{B}^{0} \bar{B}^{0}\right)(\Delta t)\right)}{N\left(B^{0} \bar{B}^{0}\right)(\Delta t)+\left(N\left(B^{0} B^{0}\right)(\Delta t)+N\left(\bar{B}^{0} \bar{B}^{0}\right)(\Delta t)\right)}=\cos \left(\Delta m_{d} \cdot \Delta t\right)
$$

where $\Delta t$ is the difference between the two neutral $B$ decay times. The simplest way to determine the $b$ quark flavor of the decaying neutral $B$ is to use primary leptons as tagging particles. $A_{\Delta m_{d}}(\Delta t)$ can be constructed directly from the rates of "like-sign" $\left(l^{+}, l^{+}\right)+\left(l^{-}, l^{-}\right)$and "unlike-sign" $\left(l^{+}, l^{-}\right)$ events. A sample of 100,000 dilepton events has been selected from 23 million $B \bar{B}$ pairs (charged and neutral). Cascade decays background $(b \rightarrow c \rightarrow l)$ is suppressed by a neural network using kinematic information of the events. The charged $B$ content of the sample is extracted from the data together with $\Delta m_{d}$. In the boost approximation used in this measurement the decay time difference is calculated as: $\Delta t=\Delta z / c<\beta \gamma>$, where the small flight path of the $B$ mesons perpendicular to the $\mathrm{z}$ axis is ignored. The $\Delta z$ resolution is extracted from simulation and has been validated by comparisons to real data control samples (e.g. $J / \psi$ decays). The time dependent asymmetry is shown in Fig. 1. The preliminary result is:

$$
\Delta m_{d}=0.499 \pm 0.010 \text { (stat.) } \pm 0.012 \text { (syst.) } \hbar \mathrm{ps}^{-1}
$$

$C P$ and $T$ violation in $B^{0} \bar{B}^{0}$ mixing can be probed in the same way as in $K^{0} \bar{K}^{0}$ mixing [4]. Its magnitude is given by the parameter $\varepsilon$ :

$$
A_{T}(|\Delta t|)=\frac{N b\left(l^{+}, l^{+}\right)-N b\left(l^{-}, l^{-}\right)}{N b\left(l^{+}, l^{+}\right)+N b\left(l^{-}, l^{-}\right)} \approx \frac{4 R e(\varepsilon)}{1+|\varepsilon|^{2}}
$$

Using our dilepton sample we obtain the preliminary result:

$$
\frac{4 R e(\varepsilon)}{1+|\varepsilon|^{2}}=(1.2 \pm 2.9(\text { stat. }) \pm 3.6(\text { syst. })) \times 10^{-3}
$$

which is the most precise measurement of this quantity to date. The $A_{T}$ distribution is also shown in Fig. 1.

\section{Time-dependent measurements with exclusively reconstructed $B$ events}

High purity $B$ event samples are obtained when the hadronic decay of one $B$ meson is fully reconstructed. In such events the kinematics and decay vertex, as well as the flavor or $C P$ content of the exclusively reconstructed $B$ are fully determined. The events where in addition the decay vertex of the other $B$ meson can be determined are used for lifetime measurements. The $B^{0} \bar{B}^{0}$ events where both the decay vertex and flavor (at decay time) of the other $B$ can be determined are used for mixing or $C P$ violation measurements. 

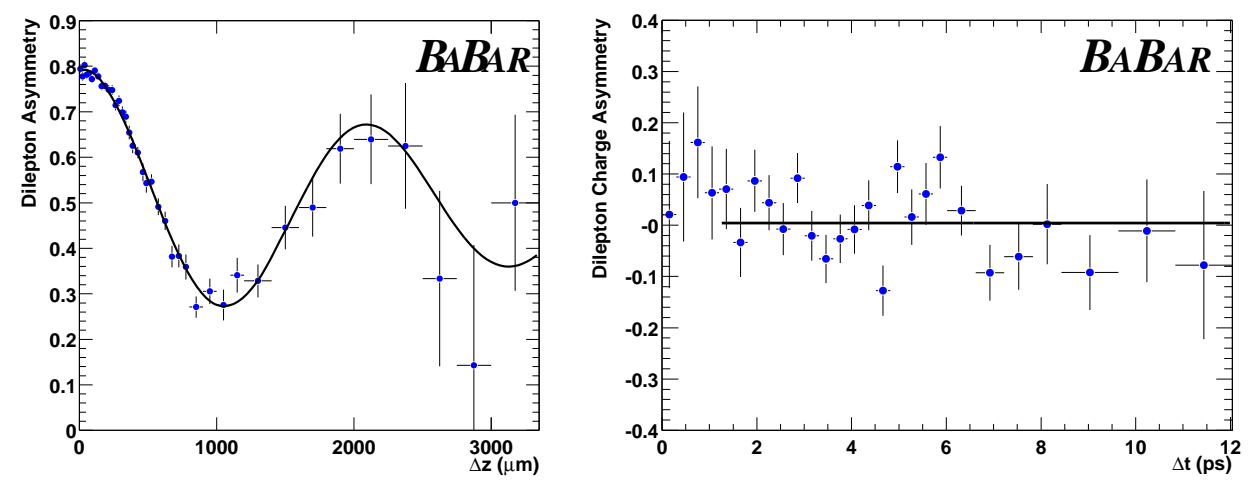

Figure 1: Left: The time dependent asymmetry between unmixed and mixed dilepton events. Right: The asymmetry $A_{T}$. The fit results are superimposed on both asymmetry plots.

\subsection{Exclusive $B$ reconstruction}

$B^{0}$ mesons are fully reconstructed in hadronic modes of (a) definite flavor: $B^{0} \rightarrow D^{(*)-} \pi^{+}, D^{(*)-} \rho^{+}$, $D^{(*)-} a_{1}^{+}, J / \psi K^{* 0}\left(K^{* 0} \rightarrow K^{+} \pi^{-}\right)$and (b) known $C P$ content: $B^{0} \rightarrow J / \psi K_{S}^{0}, \psi(2 S) K_{S}^{0}, J / \psi K_{L}^{0}$, $\chi_{c 1} K_{S}^{0}, J / \psi K^{* 0}\left(K^{* 0} \rightarrow K_{S}^{0} \pi^{0}\right)$. In the following the two samples are referred to as $B_{\text {flav }}$ and $B_{C P}$ respectively. $B^{ \pm}$mesons are reconstructed in the hadronic modes $B^{-} \rightarrow D^{0} \pi^{-}, D^{* 0} \pi^{-}, J / \psi K^{-}$, $\psi(2 S) K^{-}$(throughout this paper conjugate modes are implied).

The selections have been optimized for signal significance, using on-peak, off-peak and simulated data. Charged particle identification, mass (or mass difference) and vertex constraints are used wherever applicable. The signal for each decay mode is identified in the two-dimensional distribution of the kinematical variables $\Delta E$ and $m_{\mathrm{ES}}: \Delta E=E_{\mathrm{rec}}^{*}-E_{b}^{*}$ is the difference between the $B$ candidate energy and the beam energy and $m_{\mathrm{ES}}=\sqrt{E_{b}^{* 2}-\boldsymbol{p}_{\text {rec }}^{* 2}}$ is the mass of a particle with a reconstructed momentum $\boldsymbol{p}_{\mathrm{rec}}^{*}=\sum_{i} \boldsymbol{p}_{i}^{*}$ assumed to have the beam energy, as is the case for a true $B$ meson. In events with several $B$ candidates only the one with the smallest $\Delta E$ is considered. The $\Delta E$ and $m_{\mathrm{ES}}$ variables have minimal correlation. The resolution in $m_{\mathrm{ES}}$ is $\approx 3 \mathrm{MeV} / c^{2}$, dominated by the beam energy spread. The resolution in $\Delta E$ is mode dependent and varies in the range of $12-40 \mathrm{MeV}$. For each mode a rectangular signal region is defined by the three standard deviation bands in $m_{\mathrm{ES}}\left(5.27<m_{\mathrm{ES}}<5.29 \mathrm{GeV} / c^{2}\right)$ and $\Delta E$ (mode dependent interval). The composition of each sample is determined by fitting the $m_{\mathrm{ES}}$ distribution for candidates within the signal region in $\Delta E$ to the sum of a single Gaussian representing the signal and a background function introduced by the ARGUS collaboration [5].

\section{$5.2 \Delta t$ calculation and resolution}

Since no stable charged particle emerges from the $\Upsilon(4 S)$ decay point, the production point of the $B$ mesons and thus their individual decay times cannot be determined. However the decay time difference $\Delta t$ between the two is sufficient for the description of a coherent $B$ meson pair (decay length difference technique).

The difference of the proper decay times of the $B$ mesons $\Delta t=t_{1}-t_{2}$ is determined from the separation along the boost direction $\Delta z=z_{1}-z_{2}$, including an event-by-event correction for the 
direction of the $B$ with respect to the $z$ direction in the $\Upsilon(4 S)$ frame. $z_{1}$ is determined from the charged tracks that constitute the exclusively reconstructed $B_{1}$ candidate. The other $B$ vertex is determined by fitting the tracks not belonging to the $B_{1}$ candidate to a common vertex. Tracks from photon conversions are removed. Pairs of tracks compatible with the decay of a long lived $K^{0}$ or $\Lambda$ are replaced by the parent neutral pseudotrack. To reduce the bias in the forward $z$ direction from charm decay products, the track with the largest contribution to the vertex $\chi^{2}$, if above 6 , is removed and the fit is iterated until no track fulfills this condition. Knowledge of the beam spot location and beam direction is incorporated in the $B_{2}$ vertex determination through the addition of a pseudotrack to its vertex, computed from the $B_{1}$ vertex and three-momentum, the beam spot (with a vertical size of $10 \mu \mathrm{m}$ ) and the $\Upsilon(4 S)$ momentum. The $\Delta z$ reconstruction efficiency is $97 \%$. For $99 \%$ of the reconstructed vertices the r.m.s. $\Delta z$ resolution measured in data is $180 \mu \mathrm{m}$, dominated by the $z_{2}$ vertex. An event is accepted if it has converged fits for the two $B$ vertices, an error of less than $400 \mu \mathrm{m}$ on $\Delta z$, and a measured $|\Delta t|<20 \mathrm{ps.}$

The modelling of the resolution function $\mathcal{R}$ is a crucial element of all time-dependent measurements. In the case of $B$ lifetime measurements, studies both on simulated and real data have shown that adding a zero-mean Gaussian distribution and its convolution with a decay exponential provides the best compromise between different sources of uncertainties. The width of the Gaussian is scaled by the per-event $\Delta t$ error, derived from the vertex fits. A zero-mean Gaussian distribution with width fixed at $10 \mathrm{ps}$ is used to describe outliers (less than $1 \%$ of events with incorrectly reconstructed vertices).

In the mixing and $C P$ violation measurements the time resolution is described by the sum of three Gaussian distributions (core, tail and outliers) with different means. In the $C P$ fit the means are modeled to be proportional to the per-event $\Delta t$ error, which is correlated with the weight that the daughters of long-lived charm particles have in the tag vertex reconstruction. The core and tail widths are scaled by the per-event $\Delta t$ error. The outlier width is fixed to $8 \mathrm{ps}$.

\subsection{Flavor tagging}

For flavor tagging we exploit information from the incompletely reconstructed other $B$ decay in the event. Each event is assigned to one of four hierarchical, mutually exclusive tagging categories or excluded from further analysis. The Lepton and Kaon categories contain events with high momentum leptons from semileptonic $B$ decays or with kaons whose charge is correlated with the flavor of the decaying $b$ quark (e.g. a positive lepton or kaon yields a $B^{0}$ tag). The NT1 and NT2 categories are based on a neural network algorithm whose tagging power arises primarily from soft pions from $D^{*+}$ decays and from recovering unidentified isolated primary leptons.

The figure of merit for tagging is the effective tagging efficiency $Q_{i}=\varepsilon_{i}\left(1-2 w_{i}\right)^{2}$, where $\varepsilon_{i}$ is the fraction of events with a reconstructed tag vertex that are assigned to the $i^{\text {th }}$ category and $w_{i}$ is the mistag fraction for the same category. The statistical error on $\sin 2 \beta$ is proportional to $1 / \sqrt{Q}$, where $Q=\sum Q_{i}$. The efficiencies and mistag fractions for the four tagging categories are measured from data and summarized in Table 1 .

\section{$5.4 \quad B$ lifetime and mixing measurements}

The results presented here have been obtained from a sample of approximately 23 million $B \bar{B}$ pairs collected by $B A B A R$ between October 1999 and October 2000 . Samples of $\approx 6000 B^{0}$ and $\approx 6300 B^{+}$ signal events have been selected with background contamination of less than $10 \%$. The results of a fit with a Gaussian signal distribution and an ARGUS background function [5] are superimposed 
on the $m_{\mathrm{ES}}$ distribution of the final sample in Fig. 2. The $B$ meson lifetimes are extracted from unbinned maximum likelihood fits to the $\Delta t$ distributions, also shown in Fig. 2. We obtain:

$$
\begin{aligned}
\tau_{B^{0}} & =1.546 \pm 0.032(\text { stat }) \pm 0.022(\text { syst }) \mathrm{ps} \\
\tau_{B^{+}} & =1.673 \pm 0.032(\text { stat }) \pm 0.023(\text { syst }) \mathrm{ps} \\
\tau_{B^{+}} / \tau_{B^{0}} & =1.082 \pm 0.026(\text { stat }) \pm 0.012(\text { syst })
\end{aligned}
$$
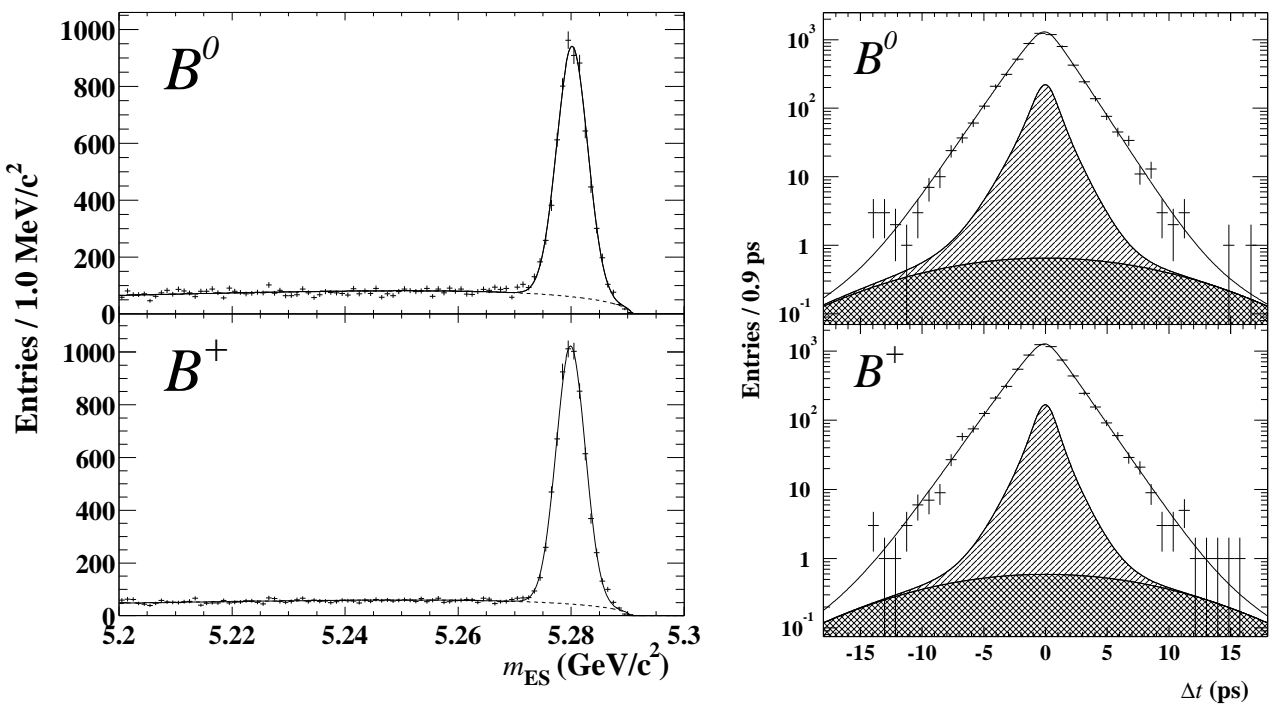

Figure 2: Left: $m_{\mathrm{ES}}$ distributions of the selected neutral (top) and charged (bottom) $B$ candidates. Right: $\Delta t$ distribution for the $B^{0}$ (top) and $B^{+}$(bottom) events within $2 \sigma$ of the $B$ mass in $m_{\mathrm{ES}}$ with superimposed fit results. The single-hatched areas are the background components and the cross-hatched areas represent the outlier contributions.

Neutral $B$ meson pairs, produced as $B^{0} \bar{B}^{0}$ decay either as $B^{0} \bar{B}^{0}$ (unmixed) or $B^{0} B^{0}\left(\bar{B}^{0} \bar{B}^{0}\right)$ (mixed), allowing to observe $B^{0} \bar{B}^{0}$ mixing and measure $\Delta m_{d}$. A sample of $\approx 4500$ such events where the flavor of the second $B$ at the time of its decay has been tagged has been obtained. The $\Delta t$ distributions and the mixing oscillation are shown in Fig. 3. From an unbinned maximum

\begin{tabular}{|l|c|c|c|c|}
\hline Tagging Category & $\varepsilon(\%)$ & $w(\%)$ & $\Delta w(\%)$ & $Q(\%)$ \\
\hline \hline Lepton & $10.9 \pm 0.3$ & $8.9 \pm 1.3$ & $0.9 \pm 2.2$ & $7.4 \pm 0.5$ \\
Kaon & $35.8 \pm 0.5$ & $17.6 \pm 1.0$ & $-1.9 \pm 1.5$ & $15.0 \pm 0.9$ \\
NT1 & $7.8 \pm 0.3$ & $22.0 \pm 2.1$ & $5.6 \pm 3.2$ & $2.5 \pm 0.4$ \\
NT2 & $13.8 \pm 0.3$ & $35.1 \pm 1.9$ & $-5.9 \pm 2.7$ & $1.2 \pm 0.3$ \\
\hline \hline all & $68.4 \pm 0.7$ & & & $26.1 \pm 1.2$ \\
\hline
\end{tabular}

Table 1: Average mistag fractions $w_{i}$ and mistag differences $\Delta w_{i}=w_{i}\left(B^{0}\right)-w_{i}\left(\bar{B}^{0}\right)$ extracted for each tagging category $i$ from the maximum-likelihood fit to the time distribution for the fullyreconstructed $B^{0}$ sample $\left(B_{\text {flav }}+B_{C P}\right)$. Uncertainties are statistical only. 
likelihood fit we obtain the preliminary result:

$$
\Delta m_{d}=0.519 \pm 0.020 \text { (stat.) } \pm 0.016 \text { (syst.) } \hbar \mathrm{ps}^{-1}
$$
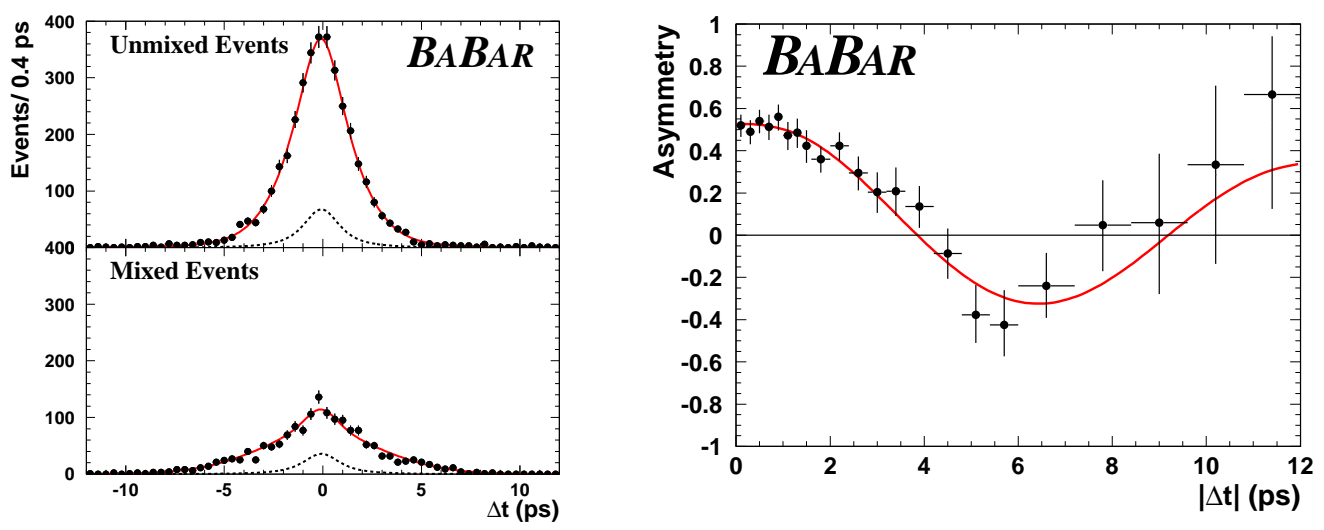

Figure 3: Left: $\Delta t$ distributions of the selected neutral unmixed (top) and mixed (bottom) events. Right: The time dependent asymmetry between unmixed and mixed events. The fit results are shown superimposed in all distributions. The dashed curves on the $\Delta t$ plots indicate the background contributions.

\section{$6 \sin 2 \beta$ and the observation of $C P$ violation}

The data set of 32 million $B \bar{B}$ pairs collected between October 1999 and May 2001 has been used to fully reconstruct a sample $B_{C P}$ of neutral $B$ mesons decaying to the $J / \psi K_{S}^{0}, \psi(2 S) K_{S}^{0}, J / \psi K_{L}^{0}$, $\chi_{c 1} K_{S}^{0}$, and $J / \psi K^{* 0}\left(K^{* 0} \rightarrow K_{S}^{0} \pi^{0}\right)$ final states. The last two modes have been added since our first $\sin 2 \beta$ publication [9]. There are several other significant changes in the analysis. Improvements in track and $K_{S}^{0}$ reconstruction efficiency in 2001 data produce a $\approx 30 \%$ increase in the yields for a given luminosity. Better alignment of the tracking systems in 2001 data and improvements in the tagging vertex reconstruction algorithm increase the sensitivity of the measurement by an additional 10\%. Optimization of the $J / \psi K_{L}^{0}$ selection increases the purity of this sample. The final $B_{C P}$ sample contains about 640 signal events and, with all the improvements, the statistical power of the analysis is almost doubled with respect to that of Ref. [9]. A sample $B_{\text {flav }}$ of 7591 fully reconstructed $B^{0}$ events in definite flavor eigenstates has been used to determine the tagging performance and a sample of 6814 fully reconstructed charged $B$ has been used for validations. Details of our samples are shown in Table 2. The samples are shown in Fig. 4 .

We examine each of the events in the $B_{C P}$ sample for evidence that the other neutral $B$ meson decayed as a $B^{0}$ or a $\bar{B}^{0}$ (flavor tag). The decay-time distributions for events with a $B^{0}$ or a $\bar{B}^{0}$ tag can be expressed in terms of a complex parameter $\lambda$ that depends on both $B^{0} \bar{B}^{0}$ mixing and on the amplitudes describing $\bar{B}^{0}$ and $B^{0}$ decay to a common final state $f$ [7]. The distribution $\mathrm{f}_{+}\left(\mathrm{f}_{-}\right)$ of the decay rate when the tagging meson is a $B^{0}\left(\bar{B}^{0}\right)$ is given by

$$
\mathrm{f}_{ \pm}(\Delta t)=\frac{\mathrm{e}^{-|\Delta t| / \tau_{B^{0}}}}{2 \tau_{B^{0}}\left(1+|\lambda|^{2}\right)} \times\left[\frac{1+|\lambda|^{2}}{2} \pm \operatorname{Im} \lambda \sin \left(\Delta m_{B^{0}} \Delta t\right) \mp \frac{1-|\lambda|^{2}}{2} \cos \left(\Delta m_{B^{0}} \Delta t\right)\right]
$$



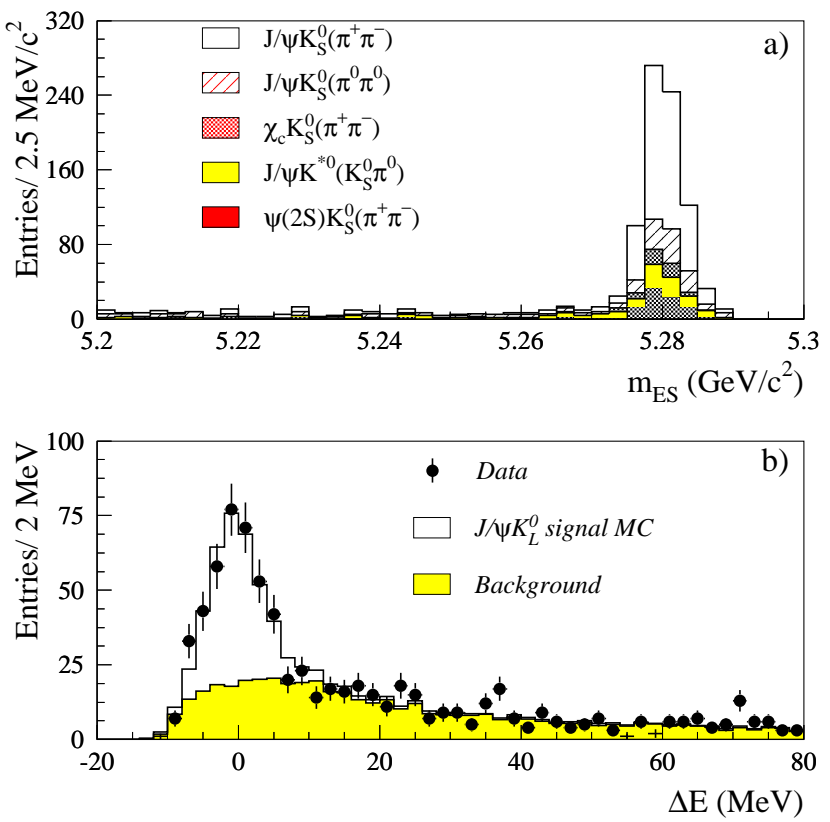

Figure 4: a) Distribution of $m_{\mathrm{ES}}$ for $B_{C P}$ candidates having a $K_{S}^{0}$ in the final state; b) distribution of $\Delta E$ for $J / \psi K_{L}^{0}$ candidates.

Table 2: Number of tagged events, signal purity and result of fitting for $C P$ asymmetries in the full $C P$ sample and in various subsamples, as well as in the $B_{\text {flav }}$ and charged $B$ control samples. Errors are statistical only.

\begin{tabular}{cccc}
\hline \hline Sample & $N_{\text {tag }}$ & Purity $(\%)$ & $\sin 2 \beta$ \\
\hline$J / \psi K_{S}^{0}, \psi(2 S) K_{S}^{0}, \chi_{c 1} K_{S}^{0}$ & 480 & 96 & $0.56 \pm 0.15$ \\
$J / \psi K_{L}^{0}\left(\eta_{f}=+1\right)$ & 273 & 51 & $0.70 \pm 0.34$ \\
$J / \psi K^{* 0}, K^{* 0} \rightarrow K_{S}^{0} \pi^{0}$ & 50 & 74 & $0.82 \pm 1.00$ \\
\hline Full $C P$ sample & 803 & 80 & $0.59 \pm 0.14$ \\
\hline \hline$J / \psi K_{S}^{0}, \psi(2 S) K_{S}^{0}, \chi_{c 1} K_{S}^{0}$ only $\left(\eta_{f}=-1\right)$ & \\
\hline$J / \psi K_{S}^{0}\left(K_{S}^{0} \rightarrow \pi^{+} \pi^{-}\right)$ & 316 & 98 & $0.45 \pm 0.18$ \\
$J / \psi K_{S}^{0}\left(K_{S}^{0} \rightarrow \pi^{0} \pi^{0}\right)$ & 64 & 94 & $0.70 \pm 0.50$ \\
$\psi(2 S) K_{S}^{0}\left(K_{S}^{0} \rightarrow \pi^{+} \pi^{-}\right)$ & 67 & 98 & $0.47 \pm 0.42$ \\
$\chi_{c 1} K_{S}^{0}$ & 33 & 97 & $2.59 \pm 0.55$ \\
\hline Lepton tags & 74 & 100 & $0.54 \pm 0.29$ \\
Kaon tags & 271 & 98 & $0.59 \pm 0.20$ \\
NT1 tags & 46 & 97 & $0.67 \pm 0.45$ \\
NT2 tags & 89 & 95 & $0.10 \pm 0.74$ \\
\hline$B^{0}$ tags & 234 & 98 & $0.50 \pm 0.22$ \\
$\bar{B}^{0}$ tags & 246 & 97 & $0.61 \pm 0.22$ \\
\hline$B_{\text {flav }}$ non-CP sample & 7591 & 86 & $0.02 \pm 0.04$ \\
\hline Charged $B$ non- $C P$ sample & 6814 & 86 & $0.03 \pm 0.04$ \\
\hline \hline
\end{tabular}


where $\Delta t=t_{C P}-t_{\mathrm{tag}}$ is the time between the two $B$ decays, $\tau_{B^{0}}$ is the $B^{0}$ lifetime and $\Delta m_{B^{0}}$ is the mass difference determined from $B^{0} \bar{B}^{0}$ mixing 8 . The first oscillatory term in Eq. 6 is due to interference between direct decay and decay after mixing, and the second term is due to direct $C P$ violation. A difference between the $B^{0}$ and $\bar{B}^{0} \Delta t$ distributions or a $\Delta t$ asymmetry for either flavor tag is evidence for $C P$ violation.

In the Standard Model $\lambda=\eta_{f} e^{-2 i \beta}$ for charmonium-containing $b \rightarrow c \bar{c} s$ decays, $\eta_{f}$ is the $C P$ eigenvalue of the state $f$ and $\beta=\arg \left[-V_{\mathrm{cd}} V_{\mathrm{cb}}^{*} / V_{\mathrm{td}} V_{\mathrm{tb}}^{*}\right]$ is an angle of the Unitarity Triangle. Thus, the time-dependent $C P$-violating asymmetry is

$$
A_{C P}(\Delta t) \equiv \frac{\mathrm{f}_{+}(\Delta t)-\mathrm{f}_{-}(\Delta t)}{\mathrm{f}_{+}(\Delta t)+\mathrm{f}_{-}(\Delta t)}=-\eta_{f} \sin 2 \beta \sin \left(\Delta m_{B^{0}} \Delta t\right),
$$

where $\eta_{f}=-1$ for $J / \psi K_{S}^{0}, \psi(2 S) K_{S}^{0}$ and $\chi_{c 1} K_{S}^{0}$ and +1 for $J / \psi K_{L}^{0}$. Due to the presence of even $(\mathrm{L}=0,2)$ and odd $(\mathrm{L}=1)$ orbital angular momenta in the $J / \psi K^{* 0}\left(K^{* 0} \rightarrow K_{S}^{0} \pi^{0}\right)$ system, there are $C P$-even and $C P$-odd contributions to the decay rate. When the angular information in the decay is ignored, the measured $C P$ asymmetry in $J / \psi K^{* 0}$ is reduced by a dilution factor $D_{\perp}=1-2 R_{\perp}$, where $R_{\perp}$ is the fraction of the $\mathrm{L}=1$ component. We have measured $R_{\perp}=(16 \pm 3.5) \%$ [6] which, after acceptance corrections, leads to an effective $\eta_{f}=0.65 \pm 0.07$ for the $J / \psi K^{* 0}$ mode.

The $B_{C P}$ and $B_{\text {flav }}$ samples are used together in the unbinned maximum likelihood fit for the extraction of $\sin 2 \beta$. A total of 45 parameters are varied in the fit, including $\sin 2 \beta$ (1), the average mistag fraction $w$ and the difference $\Delta w$ between $B^{0}$ and $\bar{B}^{0}$ mistags for each tagging category (8), parameters for the signal $\Delta t$ resolution (16), and parameters for background time dependence (9), $\Delta t$ resolution (3) and mistag fractions (8). The determination of the mistag fractions and signal $\Delta t$ resolution function is dominated by the large $B_{\text {flav }}$ sample. Background parameters are governed by events with $m_{\mathrm{ES}}<5.27 \mathrm{GeV} / c^{2}$. As a result, the largest correlation between $\sin 2 \beta$ and any linear combination of the other free parameters is only 0.13 . We fix $\tau_{B^{0}}=1.548 \mathrm{ps}$ and $\Delta m_{B^{0}}=0.472 \hbar \mathrm{ps}^{-1}[8]$. The value of $\sin 2 \beta$ and the $C P$ asymmetry in the $\Delta t$ distribution were hidden (blind analysis) following our previous publication [9], until the event selection was optimized and all other aspects of the present analysis were complete.

Figure 5 shows the $\Delta t$ distributions and $A_{C P}$ as a function of $\Delta t$ overlaid with the likelihood fit result for the $\eta_{f}=-1$ and $\eta_{f}=+1$ samples. The probability of obtaining a lower likelihood, evaluated with a parameterized simulation of a large number of data-sized experiments, is $27 \%$. Our result is:

$$
\sin 2 \beta=0.59 \pm 0.14 \text { (stat) } \pm 0.05 \text { (syst) }
$$

Repeating the fit with all parameters except $\sin 2 \beta$ fixed to their values at the global maximum likelihood, we attribute a total contribution in quadrature of 0.02 to the error on $\sin 2 \beta$ due to the combined statistical uncertainties in mistag fractions, $\Delta t$ resolution and background parameters. The dominant sources of systematic error are the parameterization of the $\Delta t$ resolution function (0.03), due in part to residual uncertainties in SVT alignment, possible differences in the mistag fractions between the $B_{C P}$ and $B_{\text {flav }}$ samples (0.03), and uncertainties in the level, composition, and $C P$ asymmetry of the background in the selected $C P$ events (0.02). The systematic errors from uncertainties in $\Delta m_{B^{0}}$ and $\tau_{B^{0}}$ and from the parameterization of the background in the $B_{\text {flav }}$ sample are small; an increase of $0.02 \hbar \mathrm{ps}^{-1}$ in the value for $\Delta m_{B^{0}}$ decreases $\sin 2 \beta$ by 0.015 .

The large sample of reconstructed events allows a number of consistency checks, including separation of the data by decay mode, tagging category and $B_{\text {tag }}$ flavor. The results of fits to these subsamples are shown in Table 2. Table 2 also shows results of fits to the samples of non- $C P$ decay 


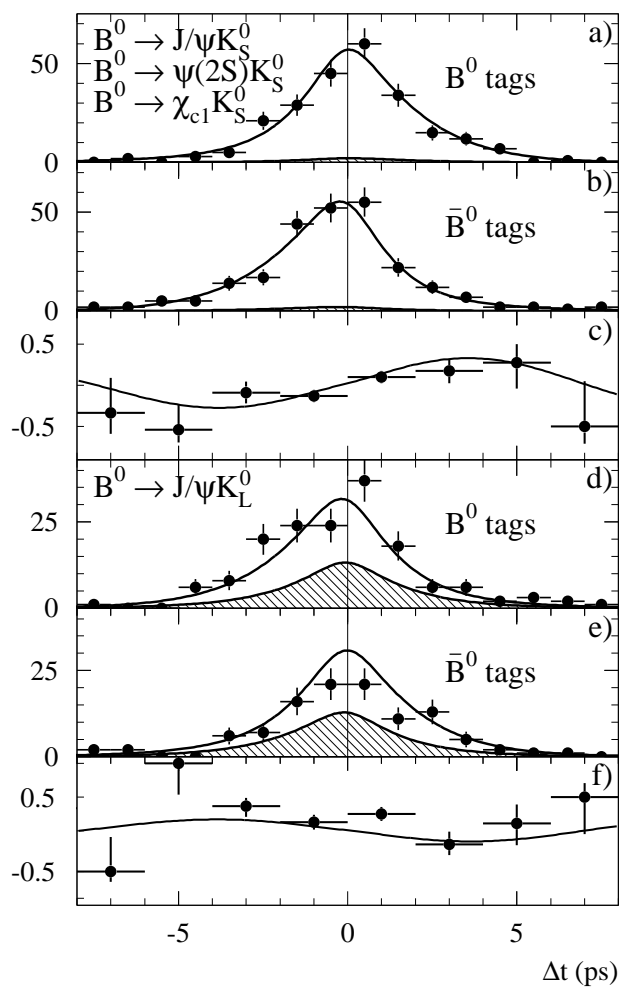

Figure 5: Number of $\eta_{f}=-1$ candidates $\left(J / \psi K_{S}^{0}, \psi(2 S) K_{S}^{0}\right.$, and $\left.\chi_{c 1} K_{S}^{0}\right)$ in the signal region a) with a $B^{0} \operatorname{tag} N_{B^{0}}$ and b) with a $\bar{B}^{0} \operatorname{tag} N_{\bar{B}^{0}}$, and c) the asymmetry $\left(N_{B^{0}}-N_{\bar{B}^{0}}\right) /\left(N_{B^{0}}+N_{\bar{B}^{0}}\right)$, as functions of $\Delta t$. The solid curves represent the result of the combined fit to all selected $C P$ events; the shaded regions represent the background contributions. Figures d)-f) contain the corresponding information for the $\eta_{f}=+1$ mode $\left(J / \psi K_{L}^{0}\right)$. The likelihood is normalized to the total number of $B^{0}$ and $\bar{B}^{0}$ tags. The value of $\sin 2 \beta$ is independent of the individual normalizations and therefore of the difference between the number of $B^{0}$ and $\bar{B}^{0}$ tags.

modes, where no statistically significant asymmetry is found. Performing the current analysis on the previously published data sample and decay modes yields a value of $\sin 2 \beta=0.32 \pm 0.18$, consistent with the published value [9]. For only these decay modes, the year 2001 data yield $\sin 2 \beta$ $=0.83 \pm 0.23$, consistent with the $1999-2000$ results at the $1.8 \sigma$ level; for the $J / \psi K_{S}^{0}\left(K_{S}^{0} \rightarrow \pi^{+} \pi^{-}\right)$ channel the consistency is at the $1.4 \sigma$ level.

If $|\lambda|$ is allowed to float in the fit to the $\eta_{f}=-1$ sample, which has high purity and requires minimal assumptions on the effect of backgrounds, the value obtained is $|\lambda|=0.93 \pm 0.09$ (stat) \pm 0.03 (syst). The sources of the systematic error in this measurement are the same as in the $\sin 2 \beta$ analysis. In this fit, the coefficient of the $\sin \left(\Delta m_{B^{0}} \Delta t\right)$ term in Eq. 6 is measured to be $0.56 \pm 0.15$ (stat) in agreement with Table 2 .

The measurement of $\sin 2 \beta=0.59 \pm 0.14$ (stat) \pm 0.05 (syst) establishes $C P$ violation in the $B^{0}$ meson system at the $4.1 \sigma$ level. This significance is computed from the sum in quadrature of the statistical and additive systematic errors. The probability of obtaining this value or higher in the absence of $C P$ violation is less than $3 \times 10^{-5}$. This direct measurement is consistent with the range implied by measurements and theoretical estimates of the magnitudes of CKM matrix 
elements [10].

\section{Conclusions and prospects}

37 years after the discovery of $C P$ violation in the Kaon system, $B A B A R$ has established $C P$ violation in the $B$ system with the measurement:

$$
\sin 2 \beta=0.59 \pm 0.14 \text { (stat) } \pm 0.05 \text { (syst) } .
$$

By the summer of $2002 B A B A R$ will have a data sample of more than 100 million $B \bar{B}$ pairs, bringing the precision on $\sin 2 \beta$ to less than 0.1 and allowing searches for other manifestations of $C P$ violation in the $B$ system.

\section{References}

[1] J.H. Christenson et al., Phys. Rev. Lett. 13, 138 (1964).

[2] N. Cabibbo, Phys. Rev. Lett. 10, 531 (1963); M. Kobayashi and T. Maskawa, Prog. Th. Phys. 49, 652 (1973).

[3] BABAR Collaboration, B. Aubert et al., SLAC-PUB-8569, hep-ex/0105044, to appear in Nucl. Instr. and Methods.

[4] CPLEAR Collabration, A. Apostolakis et al., Phys. Lett. 456, 297 (1999).

[5] ARGUS collaboration, H. Albrecht et al., Phys. Lett. 185, 218(1987).

[6] BABAR Collaboration, B. Aubert et al., SLAC-PUB-8898, hep-ex/0107049, submitted to Phys. Rev. Lett.

[7] See, for example, L. Wolfenstein, Eur. Phys. Jour. C 15, 115 (2000).

[8] Particle Data Group, D.E. Groom et al., Eur. Phys. Jour. C 15, 1 (2000).

[9] BABAR Collaboration, B. Aubert et al., Phys. Rev. Lett. 86, 2515 (2001).

[10] See, for example, F.J. Gilman, K. Kleinknecht and B. Renk, Eur. Phys. Jour. C 15, 110 (2000); for some further interpretation, with different statistical approaches, see A. Höcker et al., Eur. Phys. Jour. C 21,1 225-259 (2001) and M. Ciuchini et al., JHEP, 07(2001)013, and references therein. 